\title{
Role of abdominal CT in predicting the diagnosis of insulin resistant diabetes abbreviated title: Diagnosing insulin resistant diabetes on abdominal CT
}

\author{
Cynthia Tan ${ }^{1}$, Aruna Vade ${ }^{1}$, Jennifer Orrico ${ }^{1}$, Julius Griauzde ${ }^{1}$, Terrence C. Demos $^{1}$, \\ Alaleh Mazhari ${ }^{2}$, Mary Ann Emanuele ${ }^{2}$ \\ ${ }^{1}$ Departments of Radiology, Loyola University Medical Center, Maywood, USA; \\ Email: ctanctan@lumc.edu, avade1@lumc.edu, tdemos@lumc.edu jorrico2@lumc.edu, jgriauzde@lumc.edu \\ ${ }^{2}$ Department of Endocrinology, Loyola University Medical Center, Maywood, USA; Email: amazhari@lumc.edu, memanue@lumc.edu
}

Received 26 June 2013; revised 29 July 2013; accepted 20 August 2013

Copyright (C) 2013 Cynthia Tan et al. This is an open access article distributed under the Creative Commons Attribution License, which permits unrestricted use, distribution, and reproduction in any medium, provided the original work is properly cited.

\begin{abstract}
We report a diagnosis of diabetes type 2 suggested by diffuse omental infiltration seen on computed tomography which resolved following treatment. There have been reports of increased macrophage infiltration in the omental fat of diabetic patients which we believe is identifiable by CT. To our knowledge, the diagnosis of diabetes with CT has not yet been reported. Diabetes type 2 is commonly a clinical diagnosis. However, undiagnosed diabetic patients may present with nonspecific manifestations and CT may be ordered as part of the initial evaluation. We present a case in which diabetes type 2 was suggested by diffuse omental infiltration.
\end{abstract}

Keywords: Omental Infiltration; Diabetes

\section{INTRODUCTION}

The obesity epidemic is increasing at an alarming rate. Health complications from obesity have dramatically impacted patients' quality of life and health care. Obesity has been strongly associated with many diseases, especially insulin resistant type 2 diabetes. Patients with diabetes type 2 are defined as either not producing enough insulin or being insulin resistant. Prior to developing type 2 diabetes, most are prediabetic as determined by elevating fasting plasma glucose levels that are greater than normal $(100 \mathrm{mg} / \mathrm{dl})$ but less than $126 \mathrm{mg} / \mathrm{dl}$. By accurately identifying patients who have not yet been diag- nosed with diabetes, we may be able to provide early initiation of treatment. Type 2 diabetes has been strongly associated with central obesity [1]. Current research has shown preferential macrophage infiltration of omental fat different from subcutaneous fat in Type 2 diabetic patients [2,3]. Chronic low-grade inflammation caused by macrophage infiltration of the omentum inhibits formation of mature adipocytes alters their function [4,5]. Though much research has been done focusing on body mass index and quantification of visceral fat by CT and MR using volumetric assessment of visceral fat, there has been no study addressing the alteration in visceral fat density as shown by CT or MR imaging in patients with insulin resistant Type 2 diabetes [6,7]. We present a patient who presented to the emergency department with abdominal pain. Abdomen pelvis CT showed diffuse infiltration of omental fat and diabetes was suggested as a possibility. Subsequently, the patient was proven to have Type 2 diabetes.

\section{CASE REPORT}

A previously healthy 49-year-old white male presented to the emergency department with abdominal pain. This pain had been intermittent every day for several months and was described as needle-like with radiation to the thighs and groin. On review of systems, the patient reported urinary frequency and a 20 pound weight loss over the prior year, currently weighing 130 pounds. His BMI was 19.8. Abdomen pelvis CT with intravenous and oral contrast material showed diffuse increased density and stranding of the omentum and a $1 \mathrm{~cm}$ hypodense liver lesion (Figure 1). No other abnormalities were seen. 
Based on the finding of omental fat infiltration without any other abdominal pathology, the possibility of Type 2 diabetes was suggested. Same day laboratory results following the abdominal CT revealed elevated blood glucose (417 units) and HbA1c (11.5), which were compatible with insulin resistant diabetes (diabetes mellitus type 2). His lipid profile was normal. Autoimmune antibody titre for glutamic acid decarboxylase, islet cell and insulin antibodies was negative. Metformin (500 mg twice a day) and glipizide (5 mg daily) were started. One month later, follow up liver protocol CT to evaluate the liver lesion showed no omental infiltration and a stable hepatic hemangioma (Figure 2). Over the period of two and a half years of treatment the patient gained 32 pounds, his blood glucose level was normal and HbA1c level had dropped to 6.7.

\section{DISCUSSION}

In the US, 23.6 million adults are estimated to have diabetes. Zhang et al. have estimated that one-fourth of these patients remain undiagnosed. The National Health and Nutrition Examination Survey is a random stratified survey used to identify adults who have elevated fasting

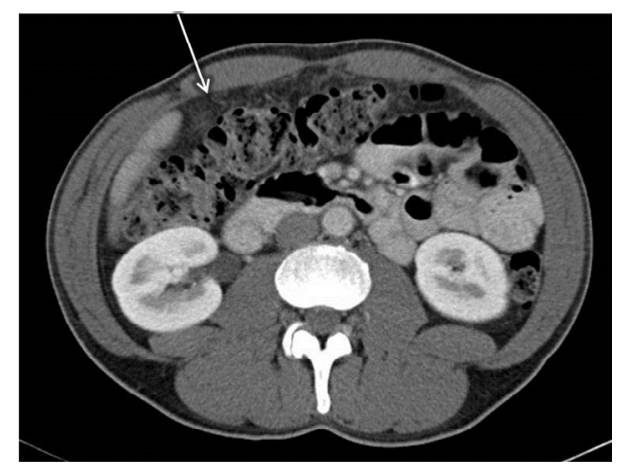

Figure 1. Contrast enhanced axial CT scan of the abdomen shows diffuse increased density and stranding of the omentum (arrow).

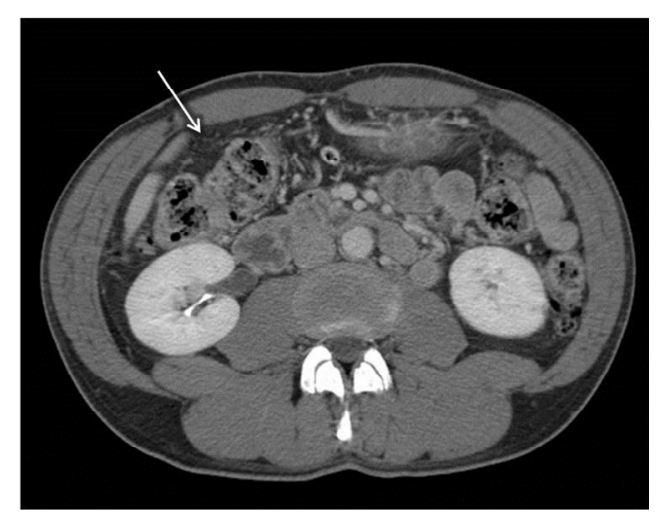

Figure 2. Contrast enhanced axial CT scan of the abdomen one month after treatment for diabetes shows no omental infiltration (arrow). plasma glucose and have never been told they have diabetes. The prevalence of undiagnosed diabetics was multiplied by the Census Bureau population in 2007. Care of known diabetics is estimated to cost $\$ 174$ billion per year in higher medical costs and lose productivity [8]. Newly diagnosed diabetics often develop complications linked to diabetes including retinopathy, proteinuria, neuropathy, arterial disease, cardiovascular disease, and coronary heart disease [8]. A claims-based study suggested costs of diabetes begins at least 8 years prior to diagnosis [8]. Since patients with undiagnosed diabetes were omitted from the estimated cost, the total expense of diabetes is likely much higher [8]. Estimated national medical costs associated with undiagnosed diabetes depend on the estimated prevalence and the ratio of per capita health care used by undiagnosed diabetics [8]. Impaired glucose tolerance has been positively correlated with obesity [9]. In obese patients, central adiposity shows a strong association with obesity-related metabolic syndromes including insulin resistant diabetes [1]. Metabolic syndromes affect $20 \%-40 \%$ of US adults and are increasing in the prevalence of making it a leading public health issue [10].

Type 1 diabetes mellitus occurs in young patients who have a lack of insulin and have normal or low BMI and a normal lipid profile [11]. Some diabetic patients have normal or low BMI, and normal lipid profile but have latent autoimmune diabetes (LADA) [12]. They present at an older age than individuals with Type 1 diabetic patients and usually need insulin after initially responding to oral medication, usually within 2 - 3 years. Type 2 diabetes mellitus typically presents at an older age and is due to insulin resistance in addition to relative insulin deficiency or relative impairment of insulin secretion [13]. However, now it is being documented in younger patients due to the rise in obesity. Type 2 diabetes mellitus patients usually have elevated BMI with other comorbidities including hypertension and dyslipidemia [13]. Maturity onset diabetes of the young (MODY) is an autosomal dominant monogenetic form of diabetes. Patients present before the age of 25 years due to a dominant genetic defect [14]. These patients do not have diabetes autoimmune antibodies. Our patient was diagnosed with Type 2 diabetes despite his normal BMI and normal lipid profile because of his negative autoimmune antibody titre and negative family history of MODY.

Visceral or central body fat consists of fat in the omentum and mesentery whereas subcutaneous fat is extraperitoneal. Visceral fat is more metabolically active than subcutaneous fat, not only in the degree of lipolytic activity but also in the release of adipokines. Current research indicates that macrophage infiltration preferentially into omental fat and not subcutaneous fat is exaggerated by central obesity and is also associated with clinical parameters of central obesity comorbidities [2,3]. 
It has been shown that macrophage infiltration of the omentum induces adipose tissue inflammation that results in dysfunction of adipose tissue by activating proinflammatory cytokines that in turn induce insulin resistance, thus linking central obesity to Type 2 diabetes. [15].

The greater omentum is a fatty peritoneal extension from the stomach covering intra-abdominal organs [16]. The omentum serves to contain disease processes, however, it may also contribute to disease spread [16]. The abdominal innervation clarifies clinical findings associated with omental abnormality. The parietal peritoneum shares its somatic innervation with the anterior abdominal musculature and skin. A diseased omentum can thus irritate the adjacent parietal peritoneum due to its close proximity as well as its mobility. The anterior abdominal wall can therefore be the site of localized or diffuse tenderness, rigidity, and guarding. These nonspecific signs and symptoms can mimic more ominous diseases such as appendicitis, diverticulitis, peptic ulcer disease, cholecystitis, and pancreatitis. Omental infiltration is a nonspecific finding seen with diseased omentum and is associated with multiple etiologies including peritoneal carcinomatosis, tuberculosis peritonitis, malignant peritoneal mesothelioma, pseudomyxoma peritonei, lymphomatosis, and cirrhosis with portal hypertension. Other conditions which can demonstrate omental infiltration include omental infarction, granuloma, hematoma, and hernia. In cases where no etiology can be identified, as in our patient, the diagnosis is idiopathic omental infiltration.

The evaluation of omental processes is primarily by CT [16]. Approximately $10 \%$ of the population are estimated to obtain a CT study during their lifetime, and a total of 75 million scans are performed annually [17]. Currently, the utilization of CT to identify or follow up idiopathic diffuse omental infiltration is not done. The patient presented here had diffuse omental infiltration indicated by the increased density of the omental fat compared to subcutaneous fat. No underlying etiology was found for this infiltration. Upon treatment for diabetes with metformin and glipizide for one month, the omental fat density returned to normal. He has responded well to this treatment and has not required insulin so far.

The CT finding of diffuse omental infiltration with no etiology should raise suspicion of insulin resistant diabetes even in patients with normal or low BMI and normal lipid profile. This can lead to earlier diagnosis and treatment with reduction of comorbidities.

\section{REFERENCES}

[1] DiPietro, L., Katz, L.D. and Nadel, E.R. (1999) Excess abdominal adiposity remains correlated with altered lipid concentrations in healthy older women. International
Journal of Obesity and Related Metabolic Disorders, 23 , 432-436. http://dx.doi.org/10.1038/sj.ijo.0800848

[2] Harman-Boehm, I., Bluher, M., Redel, H., Sion-Vardy, N., Ovadia, S., Avinoach, E., et al. (2007) Macrophage infiltration into omental versus subcutaneous fat across different populations: Effect of regional adiposity and the comorbidities of obesity. The Journal of Clinical Endocrinology \& Metabolism, 92, 2240-2247. http://dx.doi.org/10.1210/jc.2006-1811

[3] Cancello, R., Henegar, C., Viguerie, N., Taleb, S., Poitou, C., Rouault, C., et al. (2005) Reduction of macrophage infiltration and chemoattractant gene expression changes in white adipose tissue of morbidly obese subjects after surgery induced weight loss. Diabetes, 54, 2277-2286. http://dx.doi.org/10.2337/diabetes.54.8.2277

[4] Lumeng, C.N. (2010) Adipose tissue macrophages: A piece of the PAI of metabolic syndrome. Science Translational Medicine, 20, 1-3.

[5] Weisberg, S.P., McCann, D., Desai, M., Rosenbaum, M., Leibel, R.L. and Ferrante Jr., A.W. (2003) Obesity is associated with macrophage accumulation in adipose tissue. The Journal of Clinical Investigation, 112, 1796-1808.

[6] Yoshizumi, T., Nakamura, T., Yamane, M., Islam, A.H., Menju, M., Yamaskai, K., et al. (1999) Abdominal fat: Standardized technique for measurement at CT. Radiology, 211, 283-286.

[7] Taylor, G. (2007) Good fat, bad fat-Does location matter? Radiology, 242, 645-646. http://dx.doi.org/10.1148/radiol.2423061562

[8] Zhang, Y., Dall, T.M., Mann, S.E., Chen, Y., Martin, J., Moore, V., et al. (2009) The economic costs of undiagnosed diabetes. Population Health Management, 12, 95101. http://dx.doi.org/10.1089/pop.2009.12202

[9] Janssen, I., Katzmarzyk, P.T. and Ross, R. (2002) Body mass index, waist circumference, and health risk: Evidence in support of current National Institutes of Health guidelines. Archives of Internal Medicine, 162, 2074 2079. http://dx.doi.org/10.1001/archinte.162.18.2074

[10] Yoshizumi, T. ( 2007) Relationship between the criteria for metabolic syndrome and the evaluation of abdominal fat distribution measured by CT scan. Japanese Journal of Radiological Technology, 63, 276-284.

[11] Naik, R.G., Brooks-Worrell, B.M. and Palmer, J.P. (2009) Latent autoimmune diabetes in adults. The Journal of Clinical Endocrinology \& Metabolism, 94, 4635-4644. http://dx.doi.org/10.1210/jc.2009-1120

[12] Leslie, R.D., Williams, R. and Pozzilli, P. (2006) Clinical review: Type 1 diabetes and latent autoimmune diabetes in adults: One end of the rainbow. The Journal of Clinical Endocrinology \& Metabolism, 91, 1654-1659. http://dx.doi.org/10.1210/jc.2005-1623

[13] DeFronzo, R.A. and Ferrannini, E. (1991) Insulin resistance. A multifaceted syndrome responsible for NIDDM, obesity, hypertension, dyslipidemia, and atherosclerotic cardiovascular disease. Diabetes Care, 14, 173-194. http://dx.doi.org/10.2337/diacare.14.3.173

[14] Naylor, R. and Philipson, L.H. (2011) Who should have genetic testing for maturity-onset diabetes of the young? 
Clinical Endocrinology (Oxford), 75, 422-426.

[15] Lumeng, C.N. and Saltiel, A. (2007) Inflammatory links between obesity and metabolic disease. Journal of Clinical Investigation, 21, 2111-2117.

[16] Yoo, E., Kim, J.H., Kim, M.J., Yu, J.S., Chung, J.J., Yoo, H.S., et al. (2007) Greater and lesser omenta: Normal anatomy and pathologic processes. RadioGraphics, 27, 707-720. http://dx.doi.org/10.1148/rg.273065085

[17] Smith-Bindman, R. (2010) Is computed tomography safe? NEJM, 363, 1-4.

http://dx.doi.org/10.1056/NEJMp1002530 